\title{
Optimal Assignment of Off-Peak Hours to Lower Curtailments in the Distribution Network
}

\author{
Luca Merciadri, Sébastien Mathieu, Damien Ernst and Quentin Louveaux \\ University of Liège - Montefiore Institute, B-4000 Liège, Belgium
}

\begin{abstract}
We consider a price signal with two settings: offpeak tariff and on-peak tariff. Some loads are connected to specific electricity meters which allow the consumption of power only in off-peak periods. Historically, off-peak periods were located during the night and on-peak periods during the day. Changing the assignment of off-peak periods is an easy method for distribution system operators to access to the flexibility of small consumers. This solution can be implemented quickly as the infrastructure needed already exists in some countries.

We propose a mixed-integer linear model to assign optimally the off-peak hours so as to minimize a societal cost. This cost gathers together the cost of electricity, the financial losses due to energy curtailments of photovoltaic installations and the loads' wellbeing. Our model considers automatic tripping of inverters and constraints of the electrical distribution networks. Simulation results show that the new disposition of off-peak hours could reduce significantly the photovoltaic energy curtailed in the summer.
\end{abstract}

Index Terms-Mathematical programming, Optimization, Smart grids, Sustainable development.

\section{NOMENCLATURE}

This section defines the main symbols used in this paper.

\section{Sets}

$\begin{array}{ll}\mathcal{T}(d) & \text { periods of day } d \\ \mathcal{T} & \text { periods, } \mathcal{T}=\{0, \ldots, T\} \\ \mathcal{T} & \mathcal{T} \backslash\{0\} \\ \mathcal{S} & \text { nodes } \\ \mathcal{S}_{h} & \text { nodes to which houses are connected } \\ \mathcal{K} & \text { flexible loads } \\ \mathcal{G} & \text { distinct groups } \\ g(k) & \text { group } g \in \mathcal{G} \text { to which load } k \text { belongs } \\ \mathcal{K}(i) & \text { flexible loads connected to node } i \\ \mathcal{D} & \text { days of the time horizon }\end{array}$

\section{Parameters}

'pr' is a period. If the chosen timestep is $1 \mathrm{~h}, \mathrm{pr}=\mathrm{h}$.

$\begin{array}{ll}C_{i j, t} & \text { capacity of link }(i, j) \text { in period } t \\ \alpha_{t} & \text { price for } 1 \mathrm{~kW} \text { pr in period } t \\ \pi^{c} & \text { consumer's money gain having produced } 1 \mathrm{~kW} \mathrm{pr} \\ \lambda & \text { cost for a loss of } 1 \mathrm{~kW} \text { pr on the consumer side } \\ \eta_{i, t} & \text { maximum power injected at node } i \text { in period } t \\ \rho_{i, t} & \text { curtailable power production at node } i \\ & \text { in period } t \\ \zeta_{i, t} & \text { static load power consumption at node } i \\ \beta_{k, t} & \text { in period } t\end{array}$

load $k$ in period $t \geq 0$

lower bound on $s_{k, t}, t \geq 0$

upper bound on $s_{k, t}, t \geq 0$

upper bound on $s_{k, t-1}+\beta_{k, t}$

nominal power of load $k$

number of off-peak periods for

group $g$ during day $d$

daily maximum number of rate switchings

Variables

$l_{i j, t} \quad$ power going from node $i$ to node $j$ in period $t$

$n_{i, t} \quad$ power balance at node $i$ in period $t$

$r_{i, t} \quad$ curtailed quantity at node $i$ in period $t$

$s_{k, t} \quad$ state of load $k$ at the end of period $t \geq 0$

$x_{k, t} \quad$ fraction of period during which load $k$

consumes power in period $t$

$u_{g, t} \quad$ equals 1 if consumer group $g$ is off-peak in period $t, 0$ otherwise

$w_{k, t} \quad$ auxiliary variable needed in order to define $x_{k, t}$

$p_{i, t} \quad$ variable power consumed at node $i$ in period $t$

$z_{g, t} \quad$ equals 1 if there is a rate switching

between periods $t-1$ and $t$

\section{INTRODUCTION}

Several methods have been proposed in order to deal with overvoltages in distribution networks. As a mean of protection, last-resort automatic security solutions such as curtailment have been implemented. Curtailing is the tripping of an inverter of a production unit when the voltage exceeds a given threshold. This phenomenon occurs more and more with the increasing penetration of PhotoVoltaic (PV) installations. Various solutions exist to further increase the injection from renewable energy sources. One of these solutions is to modify the PV's inverters controller to provide reactive power control [1]. Batteries combined with decentralized storage strategy to provide voltage control in Low-Voltage (LV) feeders also help to reduce overvoltages [2], [3]. A centralized controller could also regulate distribution network voltages by adjusting the output of distributed generations [4]. Operating these kinds of centralized controls would be the Distribution System Operator (DSO)'s responsibility.

With the growth of PV production, DSOs are more and more considering alternatives to expensive investments in network components (i.e., lines, cables, transformers, etc.). To this end, DSOs could exploit the flexibility from consumers 
connected to their distribution network. This flexibility can be provided by market-based mechanism and be directly controlled in short-term operation of distribution networks [5]. An alternative is to modify the consumer's electricity tariff depending on the time of the day to incite consumption shifting. A comparison at the market level of these incentivebased mechanisms for load curve improvement is given in [6]. From a local perspective, this indirect mechanism has been shown to avoid congestions in the distribution network if coupled with a smart electric vehicle charging algorithm [7]. One could also modify the tariff depending not only on the time but also on the location. This leads to the notion of nodal pricing. These prices can be built up to meet global objectives such as avoiding congestion [8] or maximizing the network performance and the global welfare of all the flexible consumers [9].

In this paper, we focus on a mechanism with two types of tariffs: a cheap one called off-peak tariff, and a more expensive one referred to as on-peak tariff. Historically, offpeak periods were located during the night and on-peak periods during the day. Changing the off-peak hours is an easy method to access to the flexibility of small consumers as the infrastructure needed already exists in some countries. The off-peak signal received by the consumers is broadcasted through the distribution network by a relay located at the HighVoltage (HV)/Medium-Voltage (MV) transformer. This relay broadcasts a signal in the network which indicates the starting or the ending of the off-peak period. The signal is received by the specific electricity meters which are programmed to consume power only if tariff is off-peak. These meters are called "night-only" meters. Typical loads connected to nightonly meters are electrical storage heaters and electrical boilers. These loads are externally turned on when the meter receives the off-peak tariff signal and switched off when the tariff becomes on-peak. This paper proposes to determine an optimal assignment of off-peak periods during the day in order to optimize the flexibility of the consumption of loads consuming only in off-peak periods.

This paper is structured as follows. The problem is stated in Section II. The practical considerations are discussed in Section III. Section IV describes how loads can be modeled. The mathematical formulation of the complete problem is given in Section V. Results on a typical distribution network are provided in Section VI. Finally, Section VII concludes.

\section{Problem statement}

Consider a time horizon divided in $T$ periods, and a timestep $\bar{t}$. For one day, we therefore have $\bar{t} \times T=24$. Loads responsive to off-peak patterns are divided in groups responding to the same off-peak pattern. The aim of this paper is to determine off-peak tariff patterns, i.e to obtain vectors such that

$$
u_{g, t}=\left\{\begin{array}{ll}
1, & \text { if off-peak tariff in period } t \text { for group } g \\
0, & \text { if on-peak tariff in period } t \text { for group } g
\end{array} .\right.
$$

Problem's inputs are network data, power productions, loads' power consumptions, and loads' utilities. The set of fea- sible off-peak tariff patterns is restricted by some constraints. First, the pattern must assign a given number of off-peak hours, e.g. $9 \mathrm{~h}$ of off-peak hours and $15 \mathrm{~h}$ of on-peak hours. Second, the number of tariff switchings is bounded, because the relay sending signals to every meter quickly heats up. We also consider the impact of the pattern on the distribution network at the MV level, the power capacities of the lines and of the $\mathrm{HV} / \mathrm{MV}$ transformer.

\section{PRACTICAL IMPLEMENTATION}

This section discusses the interest and the technical requirements needed to change the assignment of off-peak hours. The figures that are present in this section are realistic figures for Belgium.

In summer, the only flexible loads are electrical boilers. In winter, electrical heaters increase consequently the flexibility of the consumption. It is legitimate to ask ourself if the flexibility available in a sunny summer hour is enough to substantially reduce curtailments. Let us assume that 70 houses are present behind an MV/LV transformer. If $8 \%$ of houses are equipped with $6 \mathrm{~kW}$ of PV panels, a maximum of $33.6 \mathrm{~kW}$ of solar production can be reached during peak sunny hours in summer. If $5 \%$ of houses are equipped with a night-only meter connected to a $3 \mathrm{~kW}$ load such as an electrical boiler, one gets around $10.5 \mathrm{~kW}$ of maximal variable power consumption by MV node. It can be reasonably assumed to have, each day, at least two hours of variable power consumption from electrical boilers in summer. A minimum static power consumption by MV node in a peak sunny hour is estimated to be $20 \mathrm{~kW}$. Shifting the consumption of the boilers to these sunny peak hours can reduce the infeed to the MV network from $13.6 \mathrm{~kW}$ to $3.1 \mathrm{~kW}$, which causes neither overvoltages nor curtailments.

Is it technically feasible to switch from a predetermined off-peak periods pattern to a dynamic one? Remote controls are already able to send off-peak signals to every belgian household [10]. The main modification to the existing implementation is the fact that off-peak hours' repartition is different than the classic off-peak pattern. This has technical implications, which are now considered briefly. There is a need for a computation platform to assign the off-peak hours optimally. Following the results of Section VI-C, little computation power is needed. Changing the off-peak hours may change the configuration of electrical heaters which were previously configured to consume power during the classical off-peak hours. Performing this configuration requires to send a technician to houses with old electrical heaters connected to night-only meters which may not be responsive to the modification of the off-peak hours. Despite this additional cost for a minority of households, this solution can be seen as a quick and easy solution that can be used for night-only meters and smart meters.

\section{LOAD MODELING}

This section proposes to model, at the MV level, the behavior of the loads consuming only in off-peak periods. These loads are typically electrical boilers and heaters. The 
model proposed in this paper is a tank model similar to the one proposed in [11]. Let us consider a flexible load $k$. This load can only consume power during off-peak periods. We assume that this load consumes either zero power or nominal power $\theta_{k}$. Flexible loads are assumed to have no starting or ending phase. The energy needs in period $t$ are given by $\beta_{k, t}$ in number of periods during which nominal power needs to be consumed. The state of the load is denoted by $s_{k, t}$. It represents the number of periods during which load $k$ needs to consume at nominal power to reach its maximal storage capacity. For instance, if load $k$ is a boiler, $s_{k, t}$ is the number of off-peak periods necessary to reach its set-point temperature in period $t$.

Note that one load might consume less energy than a full period of consumption at nominal power. This is modeled by a variable $x_{k, t} \in[0 ; 1]$ defined as the fraction of period during which load $k$ consumes power in period $t$. For example, if $x_{k, t}=\frac{1}{2}$, the power consumed by the load is defined as $x_{k, t} \theta_{k}$. Therefore, this case is modeled as consuming half power during one period. $x_{k, t}$ is mathematically defined by

$$
x_{k, t}=\min \left\{u_{g(k), t} ; s_{k, t-1}+\beta_{k, t}\right\}, \quad \forall t \in \mathcal{T}_{0}, k \in \mathcal{K} .
$$

The variable $x_{k, t}$ can be expressed by linear constraints using Observation IV.1.

Observation IV.1. The affectation $c=\min \{a ; b\}$ can be replaced by the following constraints, introducing an auxiliary binary variable w:

$$
\begin{aligned}
& c \geq a-\bar{a} w \\
& c \geq b-\bar{b}(1-w) \\
& a \leq b+\bar{a} w \\
& b \leq a+\bar{b}(1-w) \\
& c \leq a+\bar{a} w \\
& c \leq b+\bar{b}(1-w)
\end{aligned}
$$

where $\bar{a}$ and $\bar{b}$ are upper bounds on $a$ and $b$.

For $s_{k, t-1}+\beta_{k, t}$, the following upper bound can be used:

$$
M_{k}:=\overline{s_{k, t-1}+\beta_{k, t}}=\sum_{t=0}^{T} \beta_{k, t} .
$$

The state of load $k$ at the end of period $t, s_{k, t}$, is given by:

$$
s_{k, t}=s_{k, t-1}+\beta_{k, t}-x_{k, t}, \quad \forall t \in \mathcal{T}_{0}, k \in \mathcal{K},
$$

with $\beta_{k, t} \in \mathbb{R}^{+} \quad \forall k \in \mathcal{K}, t \in \mathcal{T}$. The load's state is bounded by the following inequations:

$$
\tau_{k, t} \leq s_{k, t} \leq \gamma_{k, t}, \quad \forall k \in \mathcal{K}, t \in \mathcal{T}
$$

In order to ensure that the load's state at the end of the time window does not hinder the flexibility for the following day, the following constraint is added:

$$
s_{k, T} \leq \beta_{k, 0} \quad \forall k \in \mathcal{K} .
$$

The variable power consumption at node $i$ in period $t$ is the sum of the consumptions of the flexible loads connected to node $i$ :

$$
p_{i, t}=\sum_{k \in \mathcal{K}(i)} \theta_{k} x_{k, t}, \quad \forall i \in \mathcal{S}_{h}, t \in \mathcal{T}_{0} .
$$

\section{MATHEMATICAL FORMULATION}

This section describes the mathematical formulation of the problem. First, a model of inverters' tripping at the MV level is established. Second, we detail the terms constituting the objective function. Third, the complete mixed-integer linear program is defined.

\section{A. Model of inverters' tripping}

This subsection models the tripping of PV inverters at the MV level. An inverter trips when it detects a voltage ten percent higher than the nominal voltage. At that moment, it stops injecting power into the grid. We can approximate the maximum power injected in an MV/LV transformer with the following algorithm:

while $\exists$ a node with PV that has a voltage $\geq 1.1 \mathrm{~V}_{\text {nom }}$ do

Curtail the production at the highest voltage point

Compute bus voltages using a load flow

\section{end while}

return Net power injected in the slack bus.

Fig. 1 illustrates the algorithm on a very simplified example. This maximum power injected is denoted by $\eta_{i, t}$ for node $i$ in period $t$.

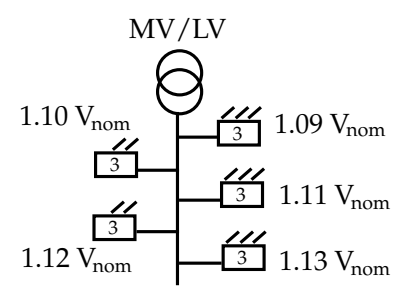

(a) Initial situation.

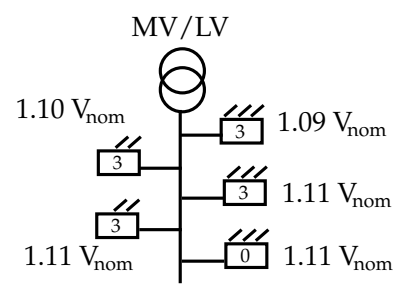

(b) One producer is curtailed.

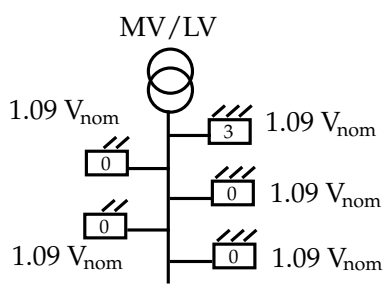

(c) $\eta_{i, t}=3 \mathrm{~kW}$

Figure 1. Example showing the application of the aforementioned algorithm.

The maximum power injected $\eta_{i, t}$ determined considering active and reactive power flows in the LV network is used as parameter in the model of the MV network. The MV network is modeled with a network flow considering only active power flow. The power balance at node $i \in \mathcal{S}_{h}$ in period $t$ is denoted by $n_{i, t}$, positive for a production. The LV part under each MV node is aggregated by three quantities: the static consumption $\zeta_{i, t}$, the variable consumption $p_{i, t}$, and the 
curtailable production $\rho_{i, t}$. The curtailment of PV panels due to overvoltage is modeled using $\eta_{i, t}$ to compute the power balance $n_{i, t}$ :

$$
n_{i, t}=\min \left\{\eta_{i, t}, \rho_{i, t}-\zeta_{i, t}-p_{i, t}\right\}, \quad \forall i \in \mathcal{S}_{h}, t \in \mathcal{T}_{0} .
$$

The minimum function models the curtailments when $\rho_{i, t}-$ $\zeta_{i, t}-p_{i, t}>\eta_{i, t}$. In this case, curtailments happen roughly from the end of the feeders to their beginnings until a value equal or just a little bit smaller than $\eta_{i, t}$. The curtailed quantity is given by

$$
r_{i, t}=\left(\rho_{i, t}-\zeta_{i, t}-p_{i, t}\right)-n_{i, t}, \quad \forall i \in \mathcal{S}_{h}, t \in \mathcal{T}_{0} .
$$

\section{B. Objective function}

The objective function represents a societal cost to minimize. This cost is divided in three parts. The first cost is an estimation of the total money loss due to curtailments. If $\pi^{c}$ is the money gain by $\mathrm{kW}$ pr produced with PV panels for a consumer, this can be expressed as

$$
\pi^{c} \sum_{i \in \mathcal{S}_{h}, t \in \mathcal{T}_{0}} r_{i, t} .
$$

The value of $\pi^{c}$ is typically the sum of the energy price and the money gain due to green certificates.

The cost of buying or selling energy using the $\alpha_{t}$ price for $1 \mathrm{~kW}$ pr in period $t$ is defined by:

$$
\sum_{i \in \mathcal{S}_{h}, t \in \mathcal{T}_{0}} \alpha_{t}\left(-n_{i, t}\right) \text {. }
$$

If $n_{i, t} \geq 0$ and $\alpha_{t}>0$, the related term is the money gain due to energy being sold.

The third cost is the cost of loads' wellbeing. This part of the objective function represents the comfort of loads' users. If $s_{k, t}=0$, the state of load $k$ is at its minimum bound at the end of period $t$. If e.g. a boiler is such that its $s_{k, t}$ is maximal, that implies that the boiler is cold. It is interesting to have $s_{k, t}$ as low as possible for every $t \in \mathcal{T}_{0}$ in order for load $k$ to be able to deal with a larger demand than foreseen. If a cost for loads' wellbeing $\lambda(€ / \mathrm{kW}$ pr) is defined, the following quantity should be minimized:

$$
\lambda \sum_{k \in \mathcal{K}, t \in \mathcal{T}_{0}} s_{k, t} \theta_{k}
$$

\section{Optimization problem}

The determination of the optimal off-peak pattern for each consumer group is given by solving the following mixedinteger linear program:

$$
\min \sum_{t \in \mathcal{T}_{0}}\left(\pi^{c} \sum_{i \in \mathcal{S}_{h}} r_{i, t}-\alpha_{t} \sum_{i \in \mathcal{S}_{h}} n_{i, t}+\lambda \sum_{k \in \mathcal{K}} s_{k, t} \theta_{k}\right)
$$

subject to

$\forall i \in \mathcal{S}, j \in \mathcal{S}, t \in \mathcal{T}_{0}$ :

$$
\begin{aligned}
& l_{i j, t} \leq C_{i j, t} \\
& l_{i j, t}+l_{j i, t}=0
\end{aligned}
$$

$\forall i \in \mathcal{S}, t \in \mathcal{T}_{0}$

$$
\sum_{j \in \mathcal{S}} l_{i j, t}=n_{i, t}
$$

$\forall i \in \mathcal{S}_{h}, t \in \mathcal{T}_{0}:$

$$
\begin{aligned}
n_{i, t} & \leq \eta_{i, t} \\
r_{i, t} & =\left(\rho_{i, t}-\zeta_{i, t}-p_{i, t}\right)-n_{i, t} \\
p_{i, t} & =\sum_{k \in \mathcal{K}(i)} \theta_{k} x_{k, t}
\end{aligned}
$$

$\forall i \in \mathcal{S}_{0} \backslash \mathcal{S}_{h}, t \in \mathcal{T}_{0}$

$$
n_{i, t}=0
$$

$\forall k \in \mathcal{K}:$

$$
\begin{array}{r}
s_{k, 0}=\beta_{k, 0} \\
s_{k, T} \leq \beta_{k, 0}
\end{array}
$$

$\forall k \in \mathcal{K}, t \in \mathcal{T}_{0}:$

$$
\begin{aligned}
s_{k, t} & =s_{k, t-1}+\beta_{k, t}-x_{k, t} \\
x_{k, t} & \geq u_{g(k), t}-w_{k, t} \\
x_{k, t} & \geq\left(s_{k, t-1}+\beta_{k, t}\right)-M_{k}\left(1-w_{k, t}\right) \\
u_{g(k), t} & \leq\left(s_{k, t-1}+\beta_{k, t}\right)+w_{k, t} \\
s_{k, t-1}+\beta_{k, t} & \leq u_{g(k), t}+M_{k}\left(1-w_{k, t}\right) \\
x_{k, t} & \leq u_{g(k), t}+w_{k, t} \\
x_{k, t} & \leq\left(s_{k, t-1}+\beta_{k, t}\right)+M_{k}\left(1-w_{k, t}\right)
\end{aligned}
$$

$\forall k \in \mathcal{K}, t \in \mathcal{T}:$

$$
\tau_{k, t} \leq s_{k, t} \leq \gamma_{k, t}
$$

$\forall g \in \mathcal{G}, d \in \mathcal{D}:$

$$
\sum_{t \in \mathcal{T}(d)} u_{g, t}=\xi_{g, d}
$$

$\forall d \in \mathcal{D}$

$$
\sum_{t \in \mathcal{T}(d)} \sum_{g \in \mathcal{G}} z_{g, t} \leq \mu
$$

$\forall t \in \mathcal{T} \backslash\{0,1\}, g \in \mathcal{G}:$

$$
\begin{aligned}
& z_{g, t} \geq u_{g, t-1}-u_{g, t} \\
& z_{g, t} \geq u_{g, t}-u_{g, t-1} \\
& z_{g, t} \leq u_{g, t-1}+u_{g, t} \\
& z_{g, t} \leq 2-u_{g, t-1}-u_{g, t} .
\end{aligned}
$$

Constraint (20) limits the power going from node $i$ to node $j$. (21)-(22) are the network flow equations for node $i$. (23) (24) define the power balance and the curtailed quantity at node $i$ in period $t$. The variable power consumed at node $i$ in period $t$ is defined by (25). (26) accounts for nodes under which no houses are present. (27) sets the initial value of the load $k$ 's state. (28) ensures that the load's state at the end of the time window does not hinder the flexibility for the following day. (29) defines the state of load $k$ at the end of period $t$. 
(30)-(35) define the fraction of period during which load $k$ consumes power in period $t$. The load's state is bounded by (36). (37) ensures a given number of off-peak periods for every day. (38)-(42) limit the number of switchings.

The variables of the problem and their respective units and domains are indicated in Table I. The total number of binary variables is of order $|\mathcal{T}|(2|\mathcal{G}|+|\mathcal{K}|)$.

Table I

VARIABLES OF THE OPTIMIZATION PROBLEM AND THEIR RESPECTIVE UNITS AND DOMAINS.

\begin{tabular}{l|ll}
\hline Variable & Unit & Domain \\
\hline$l_{i j, t}$ & $\mathrm{~kW}$ & $\mathbb{R}$ \\
$n_{i, t}$ & $\mathrm{~kW}$ & $\mathbb{R}$ \\
$r_{i, t}$ & $\mathrm{~kW}$ & $\mathbb{R}^{+}$ \\
$s_{k, t}$ & $\mathrm{pr}$ & $\mathbb{R}^{+}$ \\
$x_{k, t}$ & $\mathrm{pr}$ & {$[0 ; 1]$} \\
$u_{g, t}$ & $\mathrm{pr}$ & $\{0,1\}$ \\
$w_{k, t}$ & - & $\{0,1\}$ \\
$p_{i, t}$ & $\mathrm{~kW}$ & $\mathbb{R}^{+}$ \\
$z_{g, t}$ & $\mathrm{pr}$ & $\{0,1\}$
\end{tabular}

\section{RESULTS}

The results compare the application of the optimal assignment of off-peak hours to the classic off-peak pattern $21 \mathrm{~h}-$ $6 \mathrm{~h}$. First, we detail the parameters of the test cases. Second, we compare the classical off-peak pattern to the optimal one on a sunny summer day. Finally, the second test shows the advantages of a monthly-optimal off-peak pattern in a summer month. The solutions are obtained using CPLEX 12.6 on a computer with two Intel Core i7, $3.33 \mathrm{GHz}$ and $24 \mathrm{~GB}$ of RAM.

\section{A. Parameters}

This subsection describes the parameters that are used for the two tests. We indicate a range of values when we generate parameters using a uniform distribution. The tests are based on a typical distribution network's structure. Behind a HV/MV transformer, we consider the following structure: 12 main nodes are present behind the transformer, two nodes are connected each time to the 12 nodes. To each of these 24 nodes, three nodes are connected to a MV/LV transformer. Below each MV/LV transformer, we consider 70 houses. Four of these 70 houses are equipped with a night-only meter, each belonging to one of the four considered groups. To these night-only meters are connected an electrical boiler of nominal power $\theta \in[2.1,4.2] \mathrm{kW}$. The users' demands in $\mathrm{kWh}$ for the electrical boiler during summer are indicated in Table III. These demands should be transposed to their equivalent in periods, $\beta_{k, t}$ in function of the nominal power of the flexible load. Power production coefficients are obtained from [12] by computing ratios between hourly power production and maximal power production. Energy prices $\alpha_{t}$ are taken from [13], and static power consumptions originate from Synthetic Load Profiles values [14]. Remaining parameters are given in Table II.
Table II

ADDITIONAL PARAMETERS.

\begin{tabular}{|c|c|c|c|}
\hline \multirow{2}{*}{ Parameter } & \multicolumn{2}{|c|}{ Value } & \multirow[t]{2}{*}{ Unit } \\
\hline & VI-B & VI-C & \\
\hline$T$ & 8 & 248 & $\mathrm{pr}$ \\
\hline $\bar{t}$ & \multicolumn{2}{|c|}{3} & $\mathrm{~h} \mathrm{pr}^{-1}$ \\
\hline$\lambda, \pi^{c}$ & \multicolumn{2}{|c|}{$0.003,1.8$} & $€ / \mathrm{kW} \mathrm{pr}$ \\
\hline$\mu, \xi, \tau, \gamma$ & \multicolumn{2}{|c|}{$150,3,0,3$} & $\mathrm{pr}$ \\
\hline link capacity, HV/MV capacity & \multicolumn{2}{|c|}{20000,40000} & $\mathrm{kVA}$ \\
\hline Yearly static power consumption & \multicolumn{2}{|c|}{$[3010,5160]$} & kWh year ${ }^{-1}$ \\
\hline$\eta_{i, t} \forall i \in \mathcal{S}_{h}, t \in \mathcal{T}_{0}$ & \multicolumn{2}{|c|}{$\begin{array}{c}000000 \\
79\end{array}$} & $\mathrm{~kW}$ \\
\hline Proportion of PV & \multicolumn{2}{|c|}{30} & $\%$ \\
\hline Solar production/house with $\mathrm{PV}$ & \multirow{2}{*}{\multicolumn{2}{|c|}{$\begin{array}{c}{[4.2,6]} \\
\text { see Table III }\end{array}$}} & $\mathrm{kW}$ \\
\hline$\beta_{k, t}$ & & & $\mathrm{pr}$ \\
\hline
\end{tabular}

Table III

ELECTRICAL BOILER'S ENERGY DEMAND IN KWH.

\begin{tabular}{c|ccccccccc}
\hline$t$ & 0 & 1 & 2 & 3 & 4 & 5 & 6 & 7 & 8 \\
\hline $\bar{t} \theta \beta_{k, t}$ & 0 & 0 & 0 & 4.05 & 0 & 0 & 0.45 & 0.45 & 1.17
\end{tabular}

\section{B. Optimal off-peak pattern in a sunny summer day}

This subsection describes the results obtained on a sunny summer day: the 24th July 2012. Interesting parts of the solutions are given in Table IV. The optimization program places the off-peak hours between $12 \mathrm{~h}$ and $18 \mathrm{~h}$ and between $21 \mathrm{~h}$ and $24 \mathrm{~h}$. In particular, placing off-peak hours in the fifth period decreases curtailment costs by $44 \%$, from $504.46 €$ to $284.46 €$.

Table IV shows the power balance in each period for node 7 for which a curtailment happens in period 5 . These power balances are compared to the maximum injection through the $\mathrm{MV} / \mathrm{LV}$ transformer, $\eta_{7, t}$. When the optimal pattern is used, period 6 is off-peak, and the power balance is smaller than in the classical pattern, for which this is an on-peak period. Period 8 is an off-peak period for both settings but there is less consumption with the optimal pattern. This is a consequence of the consumption's shifting to periods 5 and 6 .

Changing the assignment of off-peak hours modifies the flow going through the the HV/MV transformer. The maximum infeed to the MV network is $15 \%$ smaller with the optimal pattern and the maximum infeed to the HV network is $5 \%$ smaller. This solution could therefore help to reduce the needs of investment in transformers.

\section{Monthly-optimal off-peak pattern in a summer month}

Instead of the classical off-peak hours assignment $21 \mathrm{~h}$ $6 \mathrm{~h}$, one could use a monthly-optimal pattern, i.e. a pattern identical for every day of the time horizon. The off-peak patterns obtained in the monthly-optimal case for July 2012 are given in Table V. They are identical for any group $g \in \mathcal{G}$. The optimization program places again the off-peak hours between $12 \mathrm{~h}$ and $18 \mathrm{~h}$ and between $21 \mathrm{~h}$ and $24 \mathrm{~h}$.

The simulation over the month provides the solutions given in Table VI. Using the monthly-optimal pattern decreases the curtailment cost by $47 \%$. Concerning the maximum infeeds, it can be seen that the monthly-optimal pattern always yields lower infeeds than the classical pattern. In particular, there is a 
Table IV

SOLUTION FOR THE SUNNY SUMMER DAY TEST.

\begin{tabular}{|c|c|c|c|c|}
\hline & \multicolumn{2}{|c|}{ Optimal pattern } & \multicolumn{2}{|c|}{ Classical pattern } \\
\hline Money gain for energy $[€]$ & \multicolumn{2}{|c|}{1021.38} & \multicolumn{2}{|c|}{1001.83} \\
\hline Curtailment cost $[€]$ & \multicolumn{2}{|c|}{284.46} & \multicolumn{2}{|c|}{504.46} \\
\hline Load wellbeing $[€]$ & \multicolumn{2}{|c|}{-2.46} & \multicolumn{2}{|c|}{-6.22} \\
\hline Welfare value $[€]$ & \multirow{2}{*}{\multicolumn{2}{|c|}{734.46}} & \multirow{2}{*}{\multicolumn{2}{|c|}{491.15}} \\
\hline Off-peak signal $\forall g \in \mathcal{G}[\mathrm{pr}]$ & & & & \\
\hline$u_{g, 1}$ & \multicolumn{2}{|c|}{0} & \multicolumn{2}{|c|}{1} \\
\hline$u_{g, 2}$ & \multicolumn{2}{|c|}{0} & \multicolumn{2}{|c|}{1} \\
\hline$u_{g, 3}$ & \multicolumn{2}{|c|}{0} & \multicolumn{2}{|c|}{0} \\
\hline$u_{g, 4}$ & \multicolumn{2}{|c|}{0} & \multicolumn{2}{|c|}{0} \\
\hline$u_{g, 5}$ & \multicolumn{2}{|c|}{1} & \multicolumn{2}{|c|}{0} \\
\hline$u_{g, 6}$ & \multicolumn{2}{|c|}{1} & \multicolumn{2}{|c|}{0} \\
\hline$u_{g, 7}$ & \multicolumn{2}{|c|}{0} & \multicolumn{2}{|c|}{0} \\
\hline$u_{g, 8}$ & \multirow{2}{*}{\multicolumn{2}{|c|}{1}} & \multirow{2}{*}{\multicolumn{2}{|c|}{1}} \\
\hline Power balance $n_{7, t}[\mathrm{~kW}]$ & & & & \\
\hline$n_{7,1}$ & -14.85 & $<\eta_{7, t}$ & -14.85 & $<\eta_{7, t}$ \\
\hline$n_{7,2}$ & -11.79 & $<\eta_{7, t}$ & -11.79 & $<\eta_{7, t}$ \\
\hline$n_{7,3}$ & 2.78 & $<\eta_{7, t}$ & 2.78 & $<\eta_{7, t}$ \\
\hline$n_{7,4}$ & 58.46 & $<\eta_{7, t}$ & 58.46 & $<\eta_{7, t}$ \\
\hline$n_{7,5}$ & 79 & $=\eta_{7, t}$ & 79 & $=\eta_{7, t}$ \\
\hline$n_{7,6}$ & 66.16 & $<\eta_{7, t}$ & 66.76 & $<\eta_{7, t}$ \\
\hline$n_{7,7}$ & 25.85 & $<\eta_{7, t}$ & 25.85 & $<\eta_{7, t}$ \\
\hline$n_{7,8}$ & -25.94 & $<\eta_{7, t}$ & -31.94 & $<\eta_{7, t}$ \\
\hline $\begin{array}{l}\text { Maximum infeed to } \\
\text { the MV network }[\mathrm{kW}]\end{array}$ & \multicolumn{2}{|c|}{2523.53} & \multicolumn{2}{|c|}{2955.53} \\
\hline $\begin{array}{l}\text { Maximum infeed to } \\
\text { the HV network }[\mathrm{kW}]\end{array}$ & \multicolumn{2}{|c|}{4662.77} & \multicolumn{2}{|c|}{4929.35} \\
\hline Time to solve [s] & \multicolumn{2}{|c|}{0.82} & \multicolumn{2}{|c|}{0.21} \\
\hline
\end{tabular}

Table V

OFF-PEAK HOUR ASSIGNMENTS FOR EVERY GROUP $g \in \mathcal{G}$, JULY 2012

\begin{tabular}{c|cccccccc}
\hline$t$ & 1 & 2 & 3 & 4 & 5 & 6 & 7 & 8 \\
\hline Classic pattern & 1 & 1 & 0 & 0 & 0 & 0 & 0 & 1 \\
Monthly-optimal pattern & 0 & 0 & 0 & 0 & 1 & 1 & 0 & 1
\end{tabular}

decrease of $14 \%$ for the maximum infeed to the MV network, and a decrease of $5 \%$ for the maximum infeed to the HV network. Notice that it takes only five minutes to solve to optimality the optimization problem on the whole month.

Table VI

SOLUTIONS OBTAINED FOR THE SUMMER MONTH TEST.

\begin{tabular}{c|rr}
\hline & Monthly-optimal pattern & Classical pattern \\
\hline $\begin{array}{c}\text { Money gain for energy } \\
\text { Curtailment cost } \\
\text { Load wellbeing } \\
\text { Welfare value }\end{array}$ & $-18909.87 €$ & $-18947.38 €$ \\
$\begin{array}{c}\text { Maximum infeed to } \\
\text { the MV network }\end{array}$ & $1135.82 €$ & $2143.68 €$ \\
$\begin{array}{c}\text { Maximum infeed to } \\
\text { the HV network } \\
\text { Time to solve }\end{array}$ & $-76.33 €$ & $-192.84 €$ \\
& $2549.03 \mathrm{~kW}$ & $2981.03 \mathrm{~kW}$ \\
& $4662.77 \mathrm{~kW}$ & $4929.35 \mathrm{~kW}$ \\
& $5 \mathrm{~min}$ & $10.07 \mathrm{~s}$
\end{tabular}

\section{CONCLUSION}

We have proposed a mixed-integer linear model to assign optimally the off-peak hours considering automatic tripping of inverters and constraints of the electrical distribution networks so as to minimize a societal cost. Simulation results show that the new disposition of off-peak hours can reduce by $50 \%$ the PV energy curtailed in the summer. The solution also helps to reduce the power flow going through the HV/MV transformer. This scheme has the main advantage of being practically implemented with very few to none investments in the current infrastructure.

Future work should focus on field testing the proposed scheme on a real part of the distribution network. Basing on DSO data, it should also be worth investigating the exact cost of the strategy that has been proposed in this paper. The proposed optimization model could be extended to include more than two different tariffs and other load models. A stochastic formulation might also be considered to assess the impact of indirect response of consumers to tariff modifications.

\section{ACKNOWLEDGMENTS}

This research is supported by the public service of Wallonia - Department of Energy and Sustainable Building within the framework of the GREDOR project. The authors thank the financial support of the Belgian Network DYSCO, an Interuniversity Attraction Poles Program initiated by the Belgian State, Science Policy Office.

\section{REFERENCES}

[1] E. Demirok, D. Sera, R. Teodorescu, P. Rodriguez, and U. Borup, "Clustered pv inverters in lv networks: An overview of impacts and comparison of voltage control strategies," in Electrical Power Energy Conference (EPEC), 2009 IEEE, Oct 2009, pp. 1-6.

[2] F. Marra, G. Yang, C. Træ holt, J. Østergaard, and E. Larsen, "A Decentralized Storage Strategy for Residential Feeders with Photovoltaics," IEEE Transactions on Smart Grids, no. 99, 2013.

[3] J. Cappelle, J. Vanalme, S. Vispoel, T. Van Maerhem, B. Verhelst, C. Debruyne, and J. Desmet, "Introducing small storage capacity at residential PV installations to prevent overvoltages," in Smart Grid Communications (SmartGridComm), 2011 IEEE International Conference on, Brussels, Belgium, 2011, pp. 534-539.

[4] T. Van Cutsem and G. Valverde, "Coordinated Voltage Control of Distribution Networks Hosting Dispersed Generation," in 22nd International Conference on Electricity Distribution, Stockholm, 2013.

[5] Q. Gemine, E. Karangelos, D. Ernst, and B. Cornélusse, "Active network management: planning under uncertainty for exploiting load modulation," in 2013 IREP Symposium-Bulk Power System Dynamics and Control -IX (IREP), Rethymnon, Greece, 2013.

[6] M. P. Moghaddam, A. Abdollahi, and M. Rashidinejad, "Flexible demand response programs modeling in competitive electricity markets," Applied Energy, vol. 88, no. 9, pp. 3257-3269, 2011.

[7] N. O'Connell, Q. Wu, S.-T. Nielsen, Arne Hejde an Cha, and J. Østergaard, "Electric Vehicle (EV) Charging Management with Dynamic Distribution System Tariff," in Innovative Smart Grid Technologies (ISGT Europe), 2011 2nd IEEE PES International Conference and Exhibition on, 2011, pp. 1-7.

[8] A. Jokic, P. van den Bosch, and R. Hermans, "Distributed, price-based control approach to market-based operation of future power systems," in Energy Market, 2009. EEM 2009. 6th International Conference on the European. IEEE, 2009, pp. 1-6.

[9] E. F. Bompard and B. Han, "Market-based control in emerging distribution system operation," Power Delivery, IEEE Transactions on, vol. 28, no. 4, pp. 2373-2382, 2013.

[10] CWaPE, "Rapport Final - REDI (Réseaux Électriques Durables et Intelligents), et Annexes. CD-12a23-CWaPE," 2012, http://www.cwape. be/docs $/ ?$ doc $=610$.

[11] S. Mathieu, D. Ernst, and Q. Louveaux, "An efficient algorithm for the provision of a day-ahead modulation service by a load aggregator," in Innovative Smart Grid Technologies Europe (ISGT EUROPE), 2013 4th IEEE/PES, Oct 2013, pp. 1-5.

[12] Elia, "Solar-PV power generation data," 2013, http://www.elia.be/ en/grid-data/power-generation/Solar-power-generation-data/Graph. Accessed on December 31, 2013.

[13] Belpex, "Belpex, the Belgian Power Exchange: Belpex. Latest Market Results." 2013, http://www.belpex.be/. Accessed on September 20, 2013.

[14] Synergrid, "Synthetic Load Profiles (SLP)," http://www.synergrid.be/. 